Cahiers $d u$ MONDE RUSSE

\section{Cahiers du monde russe}

Russie - Empire russe - Union soviétique et États indépendants

\title{
L'image du pouvoir monarchique dans les relations entre la Russie et la Pologne-Lituanie
}

Seconde moitié $\mathrm{du} \mathrm{XVI}^{\mathrm{e}}$ siècle

\section{Luc RAMOTOWSKI}

\section{(2) OpenEdition}

\section{Journals}

Édition électronique

URL : https://journals.openedition.org/monderusse/8799

DOI : 10.4000/monderusse.8799

ISSN : $1777-5388$

Éditeur

Éditions de l'EHESS

\section{Édition imprimée}

Date de publication : 1 janvier 2005

Pagination : 243-250

ISBN : 2-7132-2055-6

ISSN : $1252-6576$

Référence électronique

Luc RAMOTOWSKI, "L'image du pouvoir monarchique dans les relations entre la Russie et la Pologne-Lituanie », Cahiers du monde russe [En ligne], 46/1-2 | 2005, mis en ligne le 01 janvier 2007, consulté le 04 septembre 2022. URL : http://journals.openedition.org/monderusse/8799; DOI : https://doi.org/10.4000/monderusse.8799 
chercher : repérer : avancer

Cet article est disponible en ligne à l'adresse :

http://www.cairn.info/article.php?ID REVUE=CMR\&ID NUMPUBLIE=CMR 461\&ID ARTICLE=CMR 4610243

L'image du pouvoir monarchique dans les relations entre la Russie et la Pologne-Lituanie. Seconde moitié du XVle siècle

par Luc RAMOTOWSKI

| Editions de l'EHESS | Cahiers du monde russe

2005/1-2 - Vol 46

ISSN 1252-6576 | ISBN 2713220556 | pages 243 à 250

Pour citer cet article :

-RAMOTOWSKI L., L'image du pouvoir monarchique dans les relations entre la Russie et la Pologne-Lituanie. Seconde moitié du XVle siècle, Cahiers du monde russe 2005/ 1-2, Vol 46, p. 243-250.

Distribution électronique Cairn pour les Editions de l'EHESS.

(C) Editions de l'EHESS. Tous droits réservés pour tous pays.

La reproduction ou représentation de cet article, notamment par photocopie, n'est autorisée que dans les limites des conditions générales d'utilisation du site ou, le cas échéant, des conditions générales de la licence souscrite par votre établissement. Toute autre reproduction ou représentation, en tout ou partie, sous quelque forme et de quelque manière que ce soit, est interdite sauf accord préalable et écrit de l'éditeur, en dehors des cas prévus par la législation en vigueur en France. Il est précisé que son stockage dans une base de données est également interdit. 


\title{
L'IMAGE DU POUVOIR MONARCHIQUE DANS LES RELATIONS ENTRE LA RUSSIE ET LA POLOGNE-LITUANIE
}

\author{
Seconde moitié du XVI siècle
}

Le règne du tsar Ivan IV le Terrible est une étape importante dans la construction de l'État russe. Les contemporains ne s'y trompent pas : l'émergence de la Moscovie signifie l'apparition d'une nouvelle puissance sur l'échiquier européen. Les particularités de la culture russe les intriguent, le dynamisme de son expansion à l'ouest les inquiète, voire leur inspire de la crainte. D'où l'intérêt croissant des Occidentaux pour la découverte du monde russe. Mais la connaissance de la Moscovie passe par ses voisins les plus proches : Polonais et Lituaniens, Allemands de la Baltique, Suédois. La représentation de la Moscovie véhiculée en Europe est par conséquent influencée par le contexte politique, les rivalités ou les conflits entre voisins.

Un des principaux thèmes des ouvrages du XVI $\mathrm{I}^{\mathrm{e}}$ siècle sur la Russie concerne la monarchie moscovite, le pouvoir de son souverain et la nature même de ce pouvoir. Marshall Poe a étudié l'image du tsar russe en Europe à cette époque ${ }^{1}$. Il souligne le rôle déterminant joué par l'ambassadeur du Saint Empire, Siegmund [Sigismond] von Herberstein, dont les célèbres Rerum moscoviticarum commentarii (1556) ont été cités, voire copiés, dans nombre d'ouvrages postérieurs.

Mais c'est lorsque les nouvelles de la guerre de Livonie se diffusent en Europe que se généralise la notion de «tyrannie » pour caractériser le pouvoir d'Ivan IV. Le terme est pourtant inadapté : pour Aristote, la tyrannie est une monarchie illégitime, or les grands-princes de Moscou affirment non sans raison la légitimité de leur pouvoir et de leurs titres à régner. Le titre de tsar est justifié, il est vrai, par une prétendue filiation avec l'empereur romain Auguste, mais la volonté de s'inscrire

1. M. Poe, A People Born to Slavery, Ithaca-Londres, 2000. 
comme héritiers des empereurs byzantins, protecteurs de l'orthodoxie, prête beaucoup moins le flanc à la critique.

L'entrée des armées russes dans les États des chevaliers de l'Ordre de Livonie en 1558 amène Sigismond Auguste, roi de Pologne et grand-duc de Lituanie, à s'engager rapidement dans le conflit contre les Moscovites. Les péripéties de cette guerre, qu'il s'agisse de combats ou de négociations, multiplient les occasions de confrontation entre les systèmes de pouvoir respectifs des monarques russe et polonais.

Autre facette du conflit, la question religieuse. Les communautés urbaines allemandes de la Baltique, comme l'ensemble du monde hanséatique d'ailleurs, ont en effet accueilli favorablement la Réforme. Entre les villes allemandes de Livonie et le monarque orthodoxe, la guerre va donc se doubler d'une opposition religieuse. Dans l'ensemble du monde germanique, la conquête moscovite est dénoncée pour son extrême brutalité. Cette image est véhiculée par de nouveaux médias dans les réseaux des marchands de la Hanse. Les feuilles volantes, Flugschriften, sont diffusées dans tout l'Empire ; elles sont particulièrement riches en descriptions et représentations figurées des atrocités imputées aux Russes ${ }^{2}$. Par-delà même les pays germaniques, c'est tout le monde de la Réforme qui se sent particulièrement concerné par le conflit.

Citons pour illustrer ce propos le témoignage d'Hubert Languet. Ce protestant bourguignon est devenu diplomate au service de l'électeur de Saxe après avoir parcouru l'Europe. Entre autres, il a séjourné en Livonie peu de temps avant la conquête russe. Lors de ses différentes missions diplomatiques, il ne manque pas de s'intéresser à la politique étrangère de la Moscovie. Dans une lettre qu'il adresse en décembre 1573 à Philip Sidney alors qu'il est ambassadeur à Vienne, il redoute les succès d'Ivan IV dans la Baltique, mais n'en est pas moins impressionné par la puissance des forces moscovites :

On dit ici que le Moscovite a envahi la Lituanie avec soixante mille hommes, et qu'il est maintenant à Polock $[\ldots]^{3}$.

Languet, comme d'autres observateurs contemporains, utilise le terme de «Moscovite » pour désigner le tsar. Face à cette image négative du monarque russe qui se propage en Europe, le tsar est loin de rester silencieux : il cherche, lui aussi, à diffuser sa vérité. Et si le protestantisme naissant se passionne autant pour la guerre de Livonie, c'est aussi peut-être parce qu'Ivan IV se pose en défenseur de la «Vraie Foi » chrétienne. La guerre de Livonie est un terrain propice au tsar pour affirmer une opposition idéologique entre son État, défenseur de l'orthodoxie, et l'Occident.

2. A. Kappeler, Ivan Groznyj im Spiegel der ausländischen Druckschriften seiner Zeit, Zurich, 1969.

3. P. Sidney, H. Languet, Correspondence, Londres, 1845, p. 12 : « They say the Muscovite has invaded Lithuania with sixty thousand men, and is now at Polotz [...]». 
Les sources moscovites nous présentent une Russie qui poursuit la conquête de la Livonie dans un contexte de ferveur religieuse. Elle pense être chargée d'une mission de défense de la Vraie Foi et des images, dans sa lutte contre les iconoclastes protestants. La cause orthodoxe, thème de propagande intérieure qui sert aussi d'argument dans les négociations, est certainement utilisée pour favoriser le succès des armes russes, mais l'inverse n'est pas moins vrai : le conflit est l'occasion d'affirmer une conscience confessionnelle propre à la Russie.

C'est ainsi que la Chronique de Nikon ${ }^{4}$ présente la conquête de Polock en 1564 comme un triomphe de la foi orthodoxe. Le tsar et ses proches, les prélats de l'Église orthodoxe adressent des prières à Dieu pour la prise de la ville. Les troupes russes entrent dans la cité, précédées des icônes les plus emblématiques de l'orthodoxie russe. La victoire elle-même est entièrement relue dans une dimension théologique : Dieu punit Sigismond Auguste pour ses iniquités (l'affirmation de ses droits sur la Livonie), alors qu'Ivan IV est récompensé pour son respect de la justices.

Ivan IV n'ignore pas le succès de la pensée réformée parmi la noblesse polonaise et lituanienne. La Chronique de Nikon force le trait en décrivant une Lituanie qui a abandonné les icônes et la foi grecque ${ }^{6}$. C'est d'ailleurs à partir de la question religieuse que se développe une controverse sur la définition des pouvoirs du souverain. Le tsar moscovite ne conçoit tout simplement pas que Sigismond Auguste puisse laisser aux nobles polonais le droit d'adopter la confession de leur choix. La politique de tolérance religieuse qui prévaut alors en Pologne-Lituanie ne peut que renforcer la mauvaise opinion du tsar sur la monarchie jagellonienne, sur les relations qu'entretient le roi et grand-duc avec la noblesse. En Russie, la situation est évidemment bien différente.

Dans les États des Jagellons, la noblesse empiète de plus en plus sur le pouvoir souverain, imposant au monarque un contrôle croissant. Ivan le Terrible est parfaitement conscient des relations qui existent entre Sigismond Auguste et les Grands de la couronne. Dans sa célèbre correspondance avec le prince Kurbskij, qui le trahit durant la guerre de Livonie pour rejoindre le camp des Jagellons, le tsar s'exprime sans fard :

[...] c'est pour cela que ton esprit diabolique s'est trouvé un souverain qui ne décide de rien, mais qui reçoit des ordres de tout le monde, et qui n'ordonne rien, tel le plus vil des serviteurs?

4. Traduction anglaise : S. A. et J. B. Zenkovsky, The Nikonian Chronicle, 5 vols., Princeton, 1983-1989.

5. «Pamiatniki diplomatičeskih snošenij », RIB, t. 71, Saint-Pétersbourg, 1887, p. 358.

6. « Nikonovskaja letopis`», PSRL, t. 13, M., 2000, p. 345-346.

7. D. S. Lihačev, ed., Poslanija Ivana Groznogo, Moscou-Leningrad, 1951, p. 124. Cette première épître au prince Kurbskij a été traduite en allemand, en français, en italien ; traduction anglaise : J. L. I. Fennell, ed., The Correspondence between Prince A. M. Kurbsky and Tsar Ivan IV of Russia, 1564-1579, Cambridge, 1955. 
Un autre point fort de l'affirmation du pouvoir moscovite est le lignage. Alors qu'en Pologne et en Lituanie, l'élection du monarque par la noblesse devient de plus en plus fondamentale dans la légitimation du souverain, Ivan IV justifie son pouvoir par son appartenance à la lignée de Rurik. Cet attachement, au moins verbal, au passé kiévien lui permet en outre de revendiquer l'ensemble des pays russes du XII ${ }^{\mathrm{e}}$ siècle. Mais la mise en avant du caractère héréditaire de la fonction monarchique sert également à mettre en doute la solidité du pouvoir des Jagellons. Les ambassadeurs du tsar, fidèles à la consigne, n'hésitent pas à affirmer que le pouvoir royal polonais dépend de la seule volonté des Grands, ce qui exclut l'idée même de monarchie héréditaire :

[...] il y avait dans le pays de Lituanie des grands-princes, qui maintenant sont rois, mais ils ont reçu la royauté en dot par le mariage de la princesse royale Edwige avec Jagellon. Or cette reine n'était pas fille d'un roi polonais. Leur lignage princier était sis à Gniezno, c'est pour cela qu'ils se sont octroyé la royauté, et se sont fait appeler rois. Mais ce n'est pas une royauté qu'ils possèderaient de toute éternité, les seigneurs assoient des rois sur le trône selon leur volonté et les y gardent tant qu'ils le veulent bien ${ }^{8}$.

Le modèle de monarchie que défend Ivan IV se situe à l'opposé, comme le montre la nouvelle titulature qu'il adopte. Ivan le Terrible est en effet le premier souverain moscovite à être sacré tsar (1547), même si d'autres grands-princes ont déjà utilisé le titre antérieurement. Aux yeux des Moscovites, ce titre met Ivan IV sur un pied d'égalité avec l'empereur. C'est donc une manière efficace d'affirmer sa puissance, car il fait directement référence à une titulature byzantine. Après la chute de Constantinople et l'irruption des Ottomans en Europe, la Moscovie se trouve être désormais le seul État porteur de la Vraie Foi. Les princes de Moscou peuvent donc légitimement prétendre au titre impérial. Avec l'adoption du titre de tsar, l'horizon du pouvoir russe s'élargit: on voit apparaître dans les sources le terme de vselennaja. Il fait référence à la notion d'œkoumène chrétien. Comme l'empereur byzantin, le tsar russe pense détenir un pouvoir supérieur sur cet œkoumène. C'est dans ce cadre qu'il faut comprendre le rôle du prince danois Magnus, frère du roi Frédéric II, auquel Ivan le Terrible concède le titre de roi de Livonie, un roi soumis de manière assez souple à la Russie.

Les modèles du pouvoir souverain russe et polono-lituanien apparaissent ainsi comme antithétiques. Les groupes dirigeants ne sont pas les seuls à en prendre conscience : les simples soldats semblent avoir, eux aussi, leur opinion sur la question. Les combattants de l'un et l'autre camp qui se retrouvent au siège de Pskov n'hésitent pas à échanger leurs avis par-dessus les remparts. À partir du 24 août 1581, le nouveau roi de Pologne Etienne Bathory assiège la ville. L'événement nous est décrit dans les lettres que le jeune secrétaire Jan Piotrowski, qui se trouve sur place, envoie au grand maréchal de Pologne Andrzej Opaliński :

8. «Pamjatniki diplomatičeskih snošenij Moskovskogo gosudarstva s Pol’sko-Litovskim gosudarstvom, t. 3, 1560-1571 », Sbornik RIO, t. 71, p. 474. 
12 septembre : Les Moscovites ont pendu au mur un Hongrois au lendemain de l'assaut. Cette nuit, nous a dit Pękosławski, ils appelaient les nôtres dans les tunnels : «Vous avez vu pendre le Hongrois? nous vous pendrons tous comme ça ». Ils ont menacé quelqu'un d'autre, mais je n'ose l'écrire. «Quel genre de roi avez-vous? Il n'a ni poudre, ni argent ; venez chez nous, nous avons de la poudre, de l'argent et de tout en abondance. $»^{9}$

Le paradoxe ne saute pas tout de suite aux yeux, mais n'en est pas moins réel : même s'ils défendent des modèles monarchiques différents, les soldats des deux camps peuvent se comprendre sans intermédiaires.

La Lituanie est donc un espace de transition entre les deux sphères culturelles, entre les deux pensées politiques, un espace où le dialogue est possible. En Pologne comme en Russie, tout le monde tombe d'accord pour affirmer que la Lituanie appartient à l'héritage de la Rus' : les grands-ducs lituaniens ont soumis la partie occidentale de la principauté de Kiev. À partir du moment où la Moscovie développe son projet d'unification des terres russes, projet qui se base sur l'héritage kiévien, elle développe des prétentions territoriales sur les terres du grand-duché de Lituanie. Face à ces revendications, les Polono-Lituaniens soulignent le fait que la Moscovie n'est que l'une des principautés russes, et qu'elle ne possède pas forcément l'exclusivité de l'héritage.

Nous l'avons dit, ces échanges de points de vue sont rendus possibles par la position intermédiaire de la Lituanie. Ce sont les ambassadeurs lituaniens qui se rendent les premiers auprès d'Ivan IV au nom du roi de Pologne, c'est par le biais de ceux-ci qu'arrivent à Cracovie les informations sur la Moscovie. Ces ambassadeurs appartiennent à des familles de la noblesse ruthène orthodoxe, elles servent loyalement les Jagellons. Les plus actifs dans le dialogue diplomatique sont Wasyl Tyszkiewicz, Michał Haraburda ou encore Jerzy Chodkiewicz. Ce dernier, mort en 1569, participe à de nombreuses ambassades en Moscovie. Son frère Grzegorz, mort en 1572, est hetman des armées de Lituanie : il combat en Livonie, tout comme son neveu Jan Hieronim. Les fonctions des membres de la famille Chodkiewicz illustrent bien les liens des grandes familles de la noblesse ruthène avec le pouvoir des Jagellons.

C'est sans doute l'un des aspects les plus intéressants que nous révèlent les archives de l'Office des ambassadeurs (Posol'skij prikaz) des tsars russes : l'existence d'un dialogue au cœur duquel se trouvent ces diplomates issus de la noblesse ruthène du grand-duché.

Dans les instructions aux officiers moscovites chargés d'accueillir les ambassadeurs lituaniens, on demande parfois aux premiers de se renseigner sur la confession des ambassadeurs. L'appartenance au « rite grec » semble être un gage de confiance : lors de leur ambassade de l'été 1558, Łukasz Haraburda, le Ruthène orthodoxe, n'est pas traité de la même façon que Jan Wołoczek, Polonais catholique :

9. M. Koljakovič, éd., Dnevnik poslednego pohoda Stefana Batorija na Rossiju, Saint-Pétersbourg, 1867, p. 81 . 
Et le métropolite s'est inquiété de leur santé, il donna à Jan sa main à baiser, mais il ne le bénit pas ce jour, car il était de confession romaine, alors qu'il bénit Luc, et il leur demanda de s'asseoir ${ }^{10}$.

Les ambassadeurs lituaniens peuvent même aller jusqu'à parler directement au tsar, et non pas seulement à ses représentants. Le dialogue entre Chodkiewicz et Ivan IV est exemplaire du niveau de proximité culturelle entre ces deux personnages :

- Il est vrai que nous demeurons depuis notre jeunesse auprès de notre roi bienveillant, et ma langue russe se mêle dans mes mots avec la langue polonaise.

- Parle-nous sans aucune crainte. Les mots que tu diras en polonais, nous les comprendrons ${ }^{11}$.

Les relations avec les ambassadeurs polonais s'avèrent d'emblée plus difficiles, la distance culturelle avec les Moscovites est plus importante que pour les Lituaniens. Ils ne comprennent pas toujours bien le russe et ne sont pas forcément respectueux des coutumes locales, comme le montre le journal de l'ambassade de 1570 : certains incidents éclatent pendant le voyage jusqu'à Moscou. Dans la capitale même, l'ambassade polonaise supporte mal d'attendre près de deux semaines l'arrivée d'Ivan le Terrible. Les membres de l'ambassade tentent de franchir les murs qui entourent leurs hôtels, ils jettent des pierres à leurs gardes ${ }^{12}$.

Mais la proximité culturelle et les échanges entre les deux pouvoirs restent significatifs dans cette période, et notamment dans le contexte des élections royales de 1573 et de 1575 en Pologne-Lituanie. On voit apparaître dans le dialogue entre ambassadeurs ruthènes et pouvoir moscovite l'idée de confier les trônes polonais et lituanien à la lignée d'Ivan IV. En effet, dès 1570, l'ambassadeur Jan Skrotoszyn fait part au tsar de l'intérêt du conseil du roi de Pologne pour le fils du souverain russe : pour succéder à Sigismond Auguste, dont l'état de santé est de plus en plus préoccupant, certains avouent leur sympathie pour un futur seigneur d'origine slave ${ }^{13}$.

L'attitude ambiguë de certains magnats dans ces négociations, au rang desquels les princes Radziwiłł, reste encore à déchiffrer. Lors de l'élection de 1573, Ivan IV semble essentiellement soutenu par la petite noblesse.

La mise au pas des bojare russes peut apparaître à la petite noblesse de Pologne et de Lituanie comme une mesure encourageante. Le succès de la candidature moscovite est aussi à mettre en relation avec l'hostilité à Maximilien, le représentant des Habsbourgs parmi les prétendants au trône polonais.

L'ambassade française, qui soutient l'élection d'Henri de Valois, frère de Charles IX, a parfaitement conscience du danger que représente la candidature du

10. «Pamjatniki diplomatičeskih snošenij Moskovskogo gosudarstva s Pol’sko-Litovskim gosudarstvom, t. 2, 1533-1560 », Sbornik RIO, t. 59, p. 560.

11. Sbornik RIO, t. 71, p. 289.

12. Ibid., p. 616-758.

13. Ibid., p. 676. 
tsar. Jean de Montluc, évêque de Valence, est chargé par Catherine de Médicis de soutenir l'accession au trône de son fils Henri. Le 10 avril 1573, devant les représentants de la noblesse, il prononce à Varsovie une Harangue restée célèbre. Il y stigmatise le pouvoir du prince moscovite, le comparant à un tyran, ennemi de la chose publique, tentant ainsi d'opposer le tsar aux valeurs de la noblesse polonaise, qui puisent leurs racines dans une liberté très large accordée à cette catégorie sociale :

C'est celuy là certainement que l'on doit tenir pour un Tyran, la vie duquel est toute contaminée et souillée de meurtres, de forcemens, de pilleries et autres semblables crimes publiques : mais combien ces mœurs là sont éloignées de nostre Roy et de la manière de vivre de tous les Françoys $[\ldots]^{14}$.

Il voit dans la Pologne un rempart de la chrétienté contre les nations orientales, qualifiées de belliqueuses. Montluc utilise ce qui effraie le plus les nobles polonais dans le modèle moscovite, à savoir l'exigence d'obéissance des nobles au souverain. Ainsi, malgré une proximité culturelle, l'opposition dans la définition du pouvoir souverain resurgit.

Il conviendrait de mener une étude approfondie des discussions des diètes et des diétines préparant les élections. Cela permettrait de mieux cerner le sentiment de la noblesse polonaise et lituanienne envers le souverain moscovite, de nuancer ce sentiment en fonction de facteurs sociaux, religieux ou géographiques.

La noblesse russe s'interroge, elle aussi, sur les deux types de pouvoir monarchique que proposent Pologne et Russie. La participation des bojare à l'exercice du pouvoir souverain tend à diminuer avec la constitution de l'État russe moderne, processus qu'accélèrent encore les mesures d'Ivan IV. Les grands seigneurs russes ne peuvent qu'envier la condition des magnats polonais, la force de leurs privilèges, qui leur assurent une position dominante aussi bien dans la société que par rapport au pouvoir du souverain.

La Pologne-Lituanie est le voisin le plus proche de la Moscovie. Le domaine culturel commun aux deux pays, à savoir l'héritage de la Rus' médiévale, rend la comparaison entre les pouvoirs possible. C'est dans ce contexte qu'il faut comprendre l'attrait du modèle de gouvernement d'Ivan IV pour la masse de la petite noblesse lituanienne. À l'opposé, les bojare moscovites pourraient être tentés de se rallier au modèle que leur propose le roi de Pologne.

La suite a montré que ces deux modèles du pouvoir monarchique évoluaient dans des directions différentes. Cela n'empêchait pas de nombreuses interactions, même après la mort d'Ivan IV. Ainsi, quand Lew Sapieha, chancelier de Lituanie à partir de 1589, se rend à Moscou au printemps 1584, personne ne l'a encore averti de la mort du tsar. C'est pourtant sa seule préoccupation, comme nous le montre la 
lettre qu'il envoie au prince Radziwiłł. Celle-ci révèle également qu'il s'est informé au sujet du nouveau tsar, Fedor Ivanovič :

[...] grâce à Dieu ils n'ont pas de quoi se vanter de leur maître, j'entends dire qu'il a peu de raison, qu'il est souvent très malade. Entre les Moscovites euxmêmes, des différends et des séditions voient le jour ${ }^{15}$.

Si l'idée de la candidature d'un grand-prince de Moscou à la succession des Jagellons a pu être avancée, les principaux acteurs politiques en Pologne-Lituanie s'intéressent, eux aussi, de près aux problèmes des souverains moscovites. C'est dans ce cadre que s'inscrivent, durant le Temps des Troubles, les projets des Vasas qui cherchent à s'emparer du trône russe .

L'avènement des Romanov met un terme à l'ingérence polonaise dans les problèmes de succession en Russie. La nouvelle dynastie va réussir à consolider un pouvoir monarchique héréditaire. En Pologne-Lituanie, les monarques ont bien plus de mal à s'affirmer face aux privilèges nobiliaires. C'est peut-être l'une des clés de compréhension de la fragilité de la Pologne, qui conduira aux partages de la fin du XVIII siècle : le processus de constitution d'un État moderne semble avoir mieux réussi en Russie.

luc.ramotowski@club-internet.fr 